\title{
COMMUNICATION
}

\section{Intramolecularly stapled amphipathic peptides via a boron-sugar interaction}

Received 00th January 20xx, Accepted 00th January $20 x x$

DOI: $10.1039 / \times 0 \times x 00000 x$
Monika Kijewska*a, Angelika Czerwińska ${ }^{a}$, Samah Al Harthi ${ }^{\mathrm{b}}$, Grzegorz Wołczański ${ }^{\mathrm{a}}$, Mateusz Waliczek $^{a}$, Abdul-Hamid Emwas ${ }^{b}$, Mariusz Jaremko $^{b}$, Łukasz Jaremko ${ }^{b}$, Piotr Stefanowicz ${ }^{a}$ and Zbigniew Szewczuk
Amadori products (deoxyfructosyllysine derivatives) that can selectively interact with phenylboronic acids and borate ions were synthesized. The intramolecular interactions between the fructosyl moiety and phenylboronic acid incorporated in various positions of peptide chain were investigated using high-resolution mass spectrometry (HR-MS), circular dichroism (CD), and nuclear magnetic resonance (NMR).

The intermolecular interactions based on the reversible reaction between borate and cis-diols compounds, and the use of simple acid-base catalysed transformations, are known processes $[1,2]$. These processes are commonly used to recognize the supramolecular properties of saccharides $[3,4]$ via fluorescence[5], colorimetric[6], and electrochemical based sensors [7]. The interaction between the boron atom and the sugar residue, commonly refered to as 'stapling', is also used to stabilize peptides in an $\alpha$-helical motif via intermolecular sidechain-to-side-chain covalent bridges [8], allowing efficient competition for binding to the targets involved in proteinprotein interactions mediated by $\alpha$-helices [9]. In recent decades, several strageties have been developed in an attempt to stabilize the $\alpha$-helical structure [10]. These strageties include hydrogen bonding or electrostatic interactions between side chains, incorporation of $\alpha, \alpha$-disubstituted residues [11] such as aminoisobutyric acid [12] into the $\alpha$-helix, and the use of hydrogen-bond surrogates [13]. Side-chain crosslink formation involves an array of macrocyclization chemistries, which include hydrocarbons, triazole, lactam, thiol-based, azobenzene, and two-component double Cu-catalysed azidealkyne cycloaddition (CuAAC) staples. $[10,14]$

As reported previously $[15,16,17]$, the affinity of phenylboronic acids, such as the deoxyfructosyllysine derivatives obtained in the glycation process [18], towards Amadori products play a crucial role in the formation of

\footnotetext{
a. Faculty of Chemistry, University of Wroctaw, Joliot-Curie 14, 50-383 Wrocfaw, Poland, E-mail: monika.kijewska@chem.uni.wroc.p

b. King Abdullah University of Science and Technology (KAUST), Biological and Environmental Sciences \& Engineering Division (BESE), Thuwal, 23955-6900, Saudi Arabia.

Electronic Supplementary Information (ESI) available: See DOI: 10.1039/x0xx00000x
}

advanced glycation end products (AGEs) $[19,20]$. Notably, the AGEs have been used to enrich and isolate glycated peptides, and detect them selectively. Following these observations, we report a precise strategy for the formation of a well-designed peptide sequence containing fructosyl moiety that selectively interacts with phenylboronic acids [21] and borate ions [22].

To the best of our knowledge, there are no reports regarding the use of intramolecular stapling between phenylboronic acid and sugar residues in order to study the interactions and stability of the peptide secondary structure. Therefore, the main objective of our work was to investigate the intramolecular interactions between the fructosyl moiety and phenylboronic acid; these were incorporated into various positions on the same side of the amphipathic peptide sequence. In particular, we monitored the polypeptide secondary structure, its overall stability and conformational preferences, which may, in turn, affect non-specific binding and uptake.
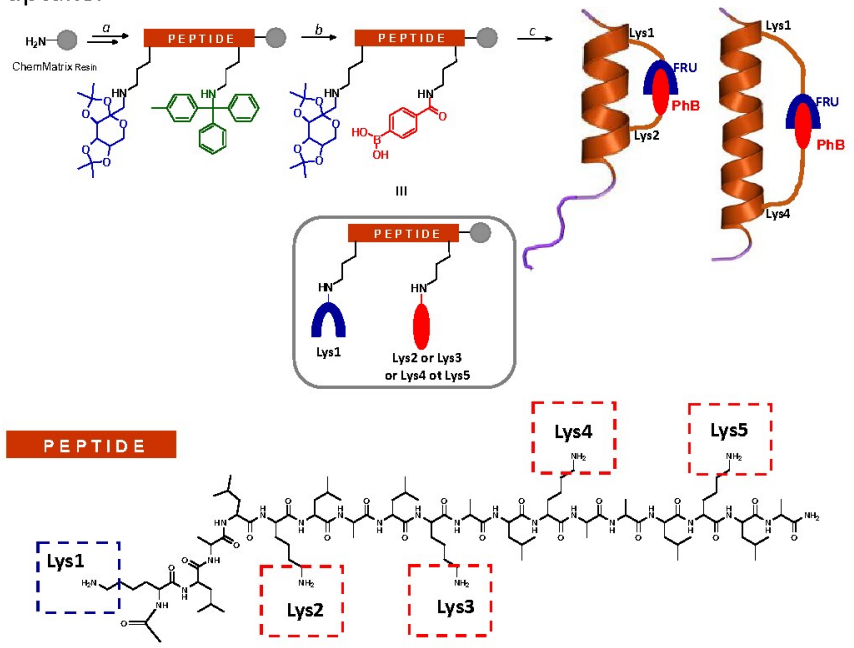

Scheme 1 Synthesis of stapled peptides of Ac-MAP analogues. Conditions: a) SPPS b) $1 \%$ TFA in DMC, PhB-OH ( 3 eq.), PyBOP (3eq.), DIEA (6 eq.) in DMF, $4 \mathrm{~h} ; \mathrm{c}$ )
TFA: $\mathrm{H}_{2} \mathrm{O}$ :TIS $(9.5: 0.25: 0.25), 8 \mathrm{~h}$.

For this purpose, we have designed and synthesized a series of $\alpha$-helical amphipathic[23] penetrating peptide (MAP) known as targeted drug carriers [24] and C-terminus peptide of RNaseA [25] analogues following the planned strategy of an 
intramolecular stapling. A series of MAP and RNaseA peptides with the sequences of Ac-KLALKLALKALKAALKLA-NH $\mathrm{NH}_{2}$ and AcEWAEKAAAKFLKAHA- $\mathrm{NH}_{2}$, respectively, and which contain the deoxyfructosyl moiety and phenylboronic acid incorporated in various positions of the peptide chain, were synthesized according to the standard Fmoc-strategy (see Scheme 1, for the synthetic details see the SI file). The modified peptides were removed from resin, lyophilized, and analyzed by ESI-MS, ESIMS/MS, LC-MS, NMR, and CD. All the MAP modified analogues, (see Table S1) that contained the deoxyfructosyl and phenylboronic acid moieties, had products with the same $\mathrm{m} / \mathrm{z}$ value of 744.815 (with a charge of $3+$ ), thus proving they have the same composition. In addition to this, the observed, characteristic isotopic distribution for the peptide with the boron atom confirms the identity of the expected products. The tandem mass spectrometry measurements are in an excellent agreement with the simulated monoisotopic mass, showing that the obtained identical monoisotopic masses correspond to cyclic products (stapled peptides). According to previous reports, boronates form esters with cis-diols, which can be hydrolyzed under acidic conditions [4]. On the contrary, the observed cyclic structures of Fru1PhB(2-5) and Fru1PhB3* form stable structures in acidic conditions. These structures include the bond between the sugar and the phenylboronic acid moieties with one blocking group located on the cis-diols of hexose moiety. Moreover, it has been found that incorporating electron-withdrawing groups into phenylboronic acid, or adding heterocyclic boronic acids to the reaction, can facilitate binding at low pH [26]. Our results showed that the acidic hydrolysis of products from resin results in the formation of irreversible intramolecular interactions between phenylboronic acid and sugar residues. For all of the obtained compounds, even after 24 hours of incubation in the released mixture, not a single blocking group was removed (see Fig. S9-S11). The uncleaved protecting group was not observed previously, as the deprotection usually occurred after about 8 hours [27]. While the ESI-MS analysis of PhB-Gly-Lys(i-Fru)- $\mathrm{NH}_{2}$ (Fig. S12) was made in methanol, the signal corresponding to deoxyfructosylated peptide with phenylboronic acid that does not form a cyclic product can be shifted because of formation of phenylboronate ester in ionization source. As observed in the ESI-MS spectrum for this model system, an additional intermolecular interaction was shown $(\mathrm{m} / \mathrm{z}=1033.502)$. In the ESI-MS spectrum of stapled peptide, a mass shift was not observed (see Fig. S13). In contrast to amphipathic peptides, model peptides form in the equilibrium of cyclic and chain forms, depending on the length of the peptide chain. In each case, however, one protecting group still remains. The ESI-MS

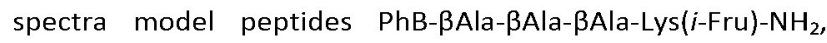

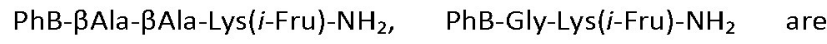
presented (Fig. S14). In the case of peptides containing two and three $\beta$ Ala residues, the low intensity signals at $m / z=602.303$ and $m / z=672.340$, respectively, of cyclic forms were observed whereas for dipeptide, only the linear form was observed. The NMR analysis of PhB-Gly-Lys(i-Fru)- $\mathrm{NH}_{2}$ confirmed the presence of one isopropylidene group (1.47 ppm and $1.35 \mathrm{ppm}$ as chemical shifts for methyl groups) (SI, NMR part). We also checked that incorporation of modifications (Fru, PhB) on different sides of the amphipathic peptide sequence results in an equilibrium between the cyclic and linear forms. The fragment of the ESI-MS spectrum of PhB-KLALKLALK(iFru)ALKAALKLA- $\mathrm{NH}_{2}$ is presented in Fig. S15. Two intensive signals corresponding to the cyclic product at $m / z=742.841$, $z=3+$, and a linear product at $m / z \quad 730.814, z=3+$ are observed. These results strongly confirm that the amphipathic sequence and presence of modifications on the same side of peptide are crucial to obtain the stable cyclic from.

All obtained peptides, modified and non-modified, were subjected to MS/MS experiments to confirm the structure of cyclic peptides. The ESI-MS/MS spectra of all peptides are shown in SI (Figs. S16-S22). All identified fragmentation ions are derived from a part of the chain that does not form a cyclic structure. In the spectra, the series of ions b are observed starting from: ion b5 at $m / z=910.512(1+)$ for Fru1PhB2, ion b9 at $m / z=1335.829(1+)$ for Fru1PhB3, ion b12 at $m / z=824.519$ (2+) for Fru1PhB4. However, for peptide Fru1PhB5, only two signals b15 and b16 were observed. For peptide Fru1PhB3*, we noticed double ions (e.g., $b_{12} y_{11}$ ), confirming the cyclic structure. In contrary to the staple peptides, the ESI-MS/MS spectrum of the non-modified peptide Ac-MAP and Ac-RNase* revealed the intensive series of ions $b$. The obtained results correlate well with literature data concerning the high stability of cyclic compounds in tandem mass spectrometry analysis [28]. To monitor the secondary structure preferences of the designed stapled peptides, we analyzed the CD and the NMR spectra. The CD spectra derived for MAP and RNase analogues showed different behavior for the obtained stapled peptides. The conformational stability of Ac-RNaseA* (AcEWAEKAAAKFLKAHA-NH ${ }_{2}$ ) and its analogue Fru1PhB3* (AcEWAEcyclo(K( $i$-Fru)AAAKFLK(PhB))AHA-NH $\mathrm{N}_{2}$ ), which contain the stapling motif between Lys- 5 and Lys- 12 residues, was analyzed by temperature-variable measurements of circular dichroism spectra in near UV. The temperature range started at $5^{\circ} \mathrm{C}$, and ended at $95^{\circ} \mathrm{C}$ for the stapled analogue. In contrast to the unmodified Ac-RnaseA*, whose CD spectrum is characteristic of peptides with an unordered conformation, and had strong negative band at about $200 \mathrm{~nm}$ measured at room temperature; the stapled analogue, Fru1PhB3* has high content of $\alpha$-helical conformation (Fig. 1).

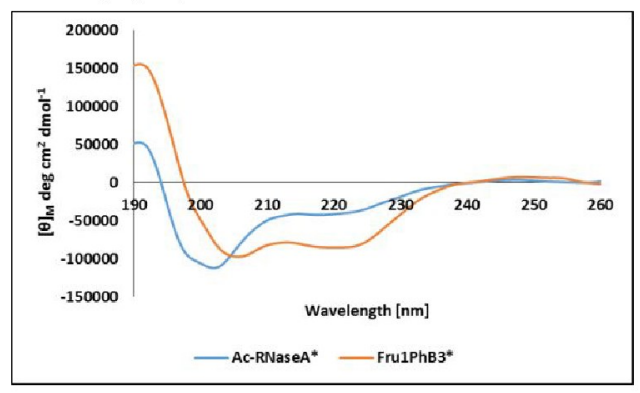

Fig. 1 Far-UV CD measurements AC-RNaseA* and stapled analog FruPhB3*

During the temperature scan from 5 to $60^{\circ} \mathrm{C}$, no significant changes in the $C D$ spectrum were observed. We found the 
melting point of the helical conformation for stapled peptide was about $65-70^{\circ} \mathrm{C}$ (Fig. S25). However, this process is accompanied by a bathochromic shifts of the negative band from $204 \mathrm{~nm}$ to $207 \mathrm{~nm}$, suggesting that changes were due to differences in conformation, and not a complete disordering of the structure. Even at temperatures higher than $90^{\circ} \mathrm{C}$, the shape of the CD spectra shows some contributions of helical and beta conformations. The linker between Lys(i) and Lys(i-7) residues in Fru1PhB3* acts as to trigger conformational changes, favoring the formation of a loop, stabilized by an antiparallel hydrogen bonds pattern in a $\beta$ hairpin-like motif, as was observed by Zhao et al.[29] The same experiments performed for MAP analogues showed different conformational preferences (Fig. S27). All peptides display two peaks around 222 and $208 \mathrm{~nm}$, which is indicative of the $\alpha$-helical structure. Following $\mathrm{CD}$ measurements, a quantitative analysis of $\mathrm{CD}$ spectra was done to predict the peptides secondary structure composition. The analysis was done using JASCO's multivariate SSE analysis program, and the results are shown in Table S2. As presented, the amount of helicity varies among of the peptides where Fru1PhB4 shows the most helical structure followed by Fru1PhB2. Fru1PhB3, Fru1PhB5, and the free peptide show more disordered structures. Afterwards, the $C D$ signal around 208 and $222 \mathrm{~nm}$ was used to monitor the thermal melting of the peptides, as they are interpreted as coming from the helical content that is melted as temperature increases. In the series of obtained derivatives, the Fru1PhB4 $(40 \pm 2 \circ \mathrm{O})$ denatures first followed by Fru1PhB5 and Fru1PhB3 (see Table S3). A free peptide and $F$ ru1PhB2 are the most stable displaying the highest Tm. Results obtained for MAP analogues presented the similar thermal stability of both unmodified peptide and cyclic analogues. We have shown that it is crucial to design peptide sequences for conformational studies of stapled peptides.

In this study, we utilized ${ }^{11} B$ NMR spectroscopy to investigate the boron interaction in staple peptides. The results show that the intensities ${ }^{11} \mathrm{~B}$ peaks observed at $18.95 \mathrm{ppm}$ correspond to the different peptides (Fru1PhB3, Fru1PhB4, and Fru1PhB5, respectively) in the investigated samples, as shown in Fig. S28. Moreover, the results show signal broadening and a slight upfield shift of sample Fru1PhB5 in comparison to the peptide-free sample PhB-Gly-Lys $(i, \mathrm{Fru})-\mathrm{NH}_{2}$, indicating that the chemical environments and/or the molecular dynamics are slightly different as shown in Fig. S29. 2D NMR analysis performed for staple peptides Fru1PhB(2-5) confirmed the presence of cyclic products that are in good agreement with data obtained from ESI-MS/MS and enzymatic hydrolysis. ${ }^{1} \mathrm{H}-{ }^{1} \mathrm{H}$ NOESY NMR spectrum for Fru1PhB2 (orange) and Fru1PhB3 (black) shows for Fru1PhB2 higher signal dispersion and more amide-amide through space ${ }^{1} \mathrm{H}-{ }^{1} \mathrm{H}$ correlations indicating the stable $\alpha$-helical structure. The Fru1PhB2 peptide has more positive carbon $\mathrm{C} \alpha$ chemical shifts and greater dispersion, pointing to its more ordered structure. Moreover, the cyclic part of the molecule has a stable $\alpha$-helical structure, while the remaining peptide chains are disordered (Fig. S30). The sugar region resonances and comparison among the overlaid spectra indicate that the predominant sugar form for Fru1PhB2 and Fru1PhB3 is different, and Fru1PhB2 shares relatively the dominant form as the PhB-Gly-Lys(i-Fru)- $\mathrm{NH}_{2}$ (Fig. S31). In the 2D HMBC spectra of Fru1PhB5 (Fig. 2) and PhB-Gly-Lys(i-Fru)$\mathrm{NH}_{2}$ (Fig. S39) the anomeric carbon (C2) region (ca. 90-110 ppm) is presented, the chemical shift (102.4 and $102.7 \mathrm{ppm}$ for Fru1PhB5 and $102.5 \mathrm{ppm}$ and $102.7 \mathrm{ppm}$ for dipeptide) indicate the dominated sugar form is $\alpha$-D-Fructofuranose (Scheme S1). The obtained chemical shits well correlate with data published by Roper et al. [30]

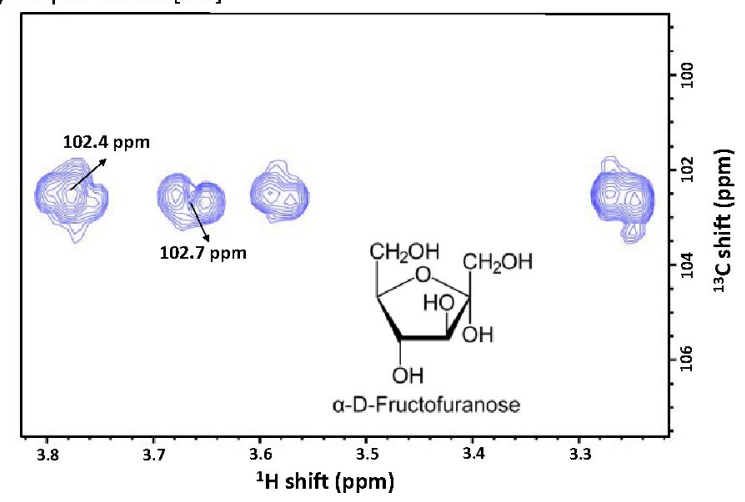

Fig. 2 HMBC spectrum of Fru1PhB5

Figure S32 highlights the sugar region (ca $60-80 \mathrm{ppm}$ for overlaid ${ }^{1} \mathrm{H}_{-}{ }^{13} \mathrm{C}$ HSQC spectra of Fru1PhB2, Fru1PhB3, and Fru1PhB4 with Fru1PhB5. We can conclude that peptide Fru1PhB4 shows a similar dominant form of sugar as Fru1PhB5. However, the peptides Fru1PhB2 and Fru1PhB3 also show the appearance of other forms. The HSQC stacked plot of the aromatic signal (ca $120-130 \mathrm{ppm}$ ) of Fru1PhB(2-5) shows different chemical shifts for different peptides indicating that the chemical environment of the $\mathrm{PhB}$ group is different from one peptide to another (Fig. S33, Table S4).

After enzymatic hydrolysis catalyzed by trypsin, the samples were analyzed by ESI-MS. The obtained staple peptides (Fru1PhB2, Fru1PhB4, Fru1PhB3, and Fru1PhB5, Fru1PhB3*) showed high stability after 24 hour of incubation and the intensive signals on the spectra belonging to the substrates were observed (Figs. S47-S55). Those isomers differ by the location of the Lys(Fru) and PhB moieties within peptide sequences. For example, peptide Fru1PhB4 produced fragments: H-Ala-Lys(i-Fru)-Leu-Ala-Leu-Lys2-OH $\quad(\mathrm{m} / z \quad=$ 816.516; $z=1+$ ), dehydrated product of H-Ala-Leu-Lys(PhB)-AlaAla-Leu-Lys5-OH $(\mathrm{m} / \mathrm{z}=844.518 ; \mathrm{z}=1+$ ), Ac-Lys(Fru)-Leu-AlaLeu-Lys2-OH $(\mathrm{m} / \mathrm{z}=776.482 ; \mathrm{z}=1+)$, H-Leu-Ala-Leu-Lys3-OH $(\mathrm{m} / \mathrm{z}$ 444.32; $\mathrm{z}=1+)$. Enzymatic hydrolysis of the Fru1PhB4 peptide occurred after the second, third, and fifth lysine residue in the sequence. The peptide chain was cleaved only at the carboxyl end of unmodified lysine, but not at lysine with a fructosyl or phenylboronic on the side chain. This observation is consistent with the previous reports indicating that the lysine modification inhibits the enzymatic activity of trypsin. [31] Analysis of the formed fragments allowed for the identification of the hydrolysis sites and confirmed the localization of Lys(Fru) and $\mathrm{Lys}(\mathrm{PhB})$ residues in the peptide chain. In the case of peptide Fru1PhB4, the cleaved at Lys 3 results in the formation of two fragments of peptides that the first one has in sequence 
the Lys(Fru), whereas the second one has in sequence Lys(PhB). The appearance of those modifications in two different peptides allowed the bond between Fru and $\mathrm{PhB}$ to dissociate, partially removing the protecting group from the hexose moiety (signal $m / z$ 776.482; $z=1+$ ), which previously was not possible (Fig. S47). Additional incubation in TFA allows an increase the intensity of the de-protected product (Fig. S48). Still, the presence of phenylboronic acid in the mixture, or the presence of another peptide containing the phenylboronic in sequence, both inhibit the removal of the protecting group from the cisdiol. For peptide Fru1PhB2 hydrolysis at Lys3 and Lys4 appeared. The two fragments were identified in the mass spectrum: cyclic product Ac-cyclo(Lys( $i$-Fru)-Leu-Ala-LeuLys(PhB))Leu-Ala-Leu-Lys3-OH at $m / z=677.420 ; z=2+$ and peptide H-Ala-Ala-Leu-Lys4-OH at $\mathrm{m} / \mathrm{z}=402.273 ; \mathrm{z}=1+$ (Fig. S39). Incubation in TFA does not cause deprotection of hexose moiety because of the presence of the cyclic form augmented by an intramolecular interaction between hexose moiety and phenyloboronic acid derivative.

In conclusion, we have presented a rational approach for the formation of stapled peptides containing phenylboronic and fructosyl moieties based on the amphipathic sequence of MAP peptide and C-terminal fragment of RNaseA. We noticed that, of crucial importance, the positions of modifications incorporated into the side chain of lysine moieties and after removing peptide from resin results in stable (in acidic condition) intramolecular interaction between Fru and PhB. We showed the impact of stapled peptides on the enzymatic stability and conformation preferences of molecules. As documented in the $C D$ experiments, we proved that the positions of the sugar and phenylboronic acid moieties in the peptide sequence, as well as the design of polypeptide sequences, influence the conformational preferences and thermal stability of formed stapled peptides. Our results showed that Fru1PhB4 exhibits the most helical structure among all synthesized analogs of MAP peptide, but is less stable than a non-modified peptide. In contrast to the unmodified AcRNaseA*, whose CD spectrum is characteristic of peptides with an unordered conformation, the stapled analogue, Fru1PhB3*, has a high content of $\alpha$-helical conformation stable at higher temperatures. Further experiments to extending the $f$ applicability and to explore potential intermolecular and intermolecular interactions between different sugar and boronic acid derivatives are currently underway in our laboratory.

\section{Acknowledgments}

This work was supported by Grant No.UMO2015/19/B/ST5/00659 from the National Science Centre, Poland. The authors would like to thank Andrzej Reszka (ShimPol, Poland) for providing the Shimadzu LCMS-IT-TOF. LJ, MJ, SMA, and A-HE would like to thank the King Abdullah University of Science and Technology (KAUST) for financial support.

\section{Conflicts of interest}

There are no conflicts to declare.

\section{Notes and references}

1 Z. Liu, H. He, Acc. Chem. Res., 2017, 50, 2185.

2 J. Boeseken, Adv. Carbohydr. Chem., 1949, 4, 189.

3 James T.D., Phillips M.D., Shinkai S. Bronic acids in saccharide recognition. The Royal Society of Chemistry 2006.

4 Y. Kanekiyoa, S. Shinkai, CHAPTER 1: Supramolecular Chemistry of Boronic Acids, in Boron: Sensing, Synthesis and Supramolecular Self-Assembly, The Royal Society of Chemistry, 2015.

5 L. Chen, E. Hwang, J. Zhang, Sensors, 2018, 18, 1440.

6 Y. Egawa, M. Ryotaro, T. Seki, Materials, 2014, 7, 1201.

7 Y. Egawa, T. Seki, S. Takahashi, J. Anzai, Mater. Sci. Eng. C, 2011, 31, 1257.

8 Y. H. Lau, P. de Andrade, Y. Wua, D. R. Spring, Chem. Soc. Rev., 2015, 44, 91.

9 J. legre, N.S. Ahmed, J. S. Gaynord, Y. Wu, K. M. Herlihy, Y. S. Tan, M.E. Lopes-Pires, R. Jha, Y. H. Lau, H. F. Sore, C. Verma, D. H. O' Donovan, N. Pugh, D. R. Spring, Chem. Sci., 2018, 25, 4638.

10 A. M. Ali, J. Atmaj, N. Van Oosterwijk, M.R. Groves, A. Dömling, Comput. Struct. Biotechnol. J. 2019, 19, 263.

11 P. Balaram, Curr. Opin. Struct. Biol., 1992, 2, 845.

12 I. L. Karle, J. L. Flippen-Anderson, K. Uma, P. Balaram, Proteins, 1990, 7, 62.

13 A. Patgiri, A. L. Jochim, P. S. Arora, Acc. Chem. Res., 2008, 41, 1289.

14 M. Scrima A. Le Chevalier-Isaad, P. Rovero, A. M. Papini, M. Chorev, A. M. D'Ursi, Eur. J. Org. Chem., 2010, 3, 446.

15 M. Kijewska, F. Nuti, M. Wierzbicka, M. Waliczek, P. Ledwoń, A. Staśkiewicz, F. Real-Fernandez, G. Sabatino, P. Rovero, P. Stefanowicz, Z. Szewczuk, A. M. Papini, Molecules, 2020, 25, $755 / 1$.

16 A. Soboleva, M. Modzel, A. Didio, H. Płóciennik, M. Kijewska, T. Grischina, T. Karonova, T. Bilova, V. Stefanov, P. Stefanowicz, A. Frolov, Anal. Methods, 2017, 9, 409.

17 A. Frolov, R. Hoffmann, R. Anal. Bioanal. Chem., 2010, 397, 2349.

18 S. D’Aronco, S. Crotti, M. Agostini, P. Traldi, N. C. Chilelli, A. Lapolla, Mass Spectrom. Rev., 2018, 9999, 1.

19 P. Ulrich, A. Cerami, Recent. Prog. Horm. Res., 2001, 56, 1.

20 P. J. Thornalley, Ann N Y Acad. Sci., 2005, 1043, 111.

21 M. Kijewska, A. Kuc, A. Kluczyk, M. Waliczek, A. ManKupisinska, J. Łukasiewicz, P. Stefanowicz, Z. Szewczuk, J. Am. Soc. Mass Spectrom., 2014, 25, 966.

22 M. Kijewska, A. Kluczyk, P. Stefanowicz, Z. Szewczuk, Rapid Commun. Mass Sp., 2009, 23, 4038.

23 J. Oehlke, A. Scheller, B. Wiesner, E. Krause, M. Beyermann, E. Klauschenz, M. Melzig, M. Bienert, Biochim. Biophys. Acta, 1998, 1414, 127.

24 M. Delcroix, L. W. Riley, Pharmaceuticals, 2010, 3, 448.

25 C.E. Schafmeister, J. Po, G.L. Verdine. J. Am. Chem. Soc., 2000; 122, 5891-5892.

26 D. Li, Q. Li, S. Wang, J. Ye, H. Nie, Z. Liu, J. Chromatogr. A, 2014, 1339, 103.

27 P. Stefanowicz, M. Kijewska, K. Kapczyńska, Z. Szewczuk, Amino Acids, 2010, 38, 881.

28 B.G. Keevil, D.P. Tierney, D.P Cooper, M.R. Morris, Clin. Chem., 2002, 48, 69.

29 B. Zhao, D. Yang, J. Ho Wong, J. Wang, C. Yin, Y. Zhu, S. Fan, T. Bun Ng, J. Xia, Z. Li. Chem. Biochem., 2016, 17(15), 1416-1420.

30 H. Roper, S. Roper, K. Heyns, Carbohydrate Res., 1983, 116, 183.

31 P. Stefanowicz, M. Kijewska, A. Kluczyk, Z. Szewczuk, Anal. Biochem., 2010, 400, 237. 